1094

\section{CORD BLOOD BRAIN DERIVED NEUROTROPHIC FACTOR: DIAGNOSTIC AND PROGNOSTIC MARKER IN FULLTERM NEWBORNS WITH PERINATAL ASPHYXIA}

doi:10.1136/archdischild-2012-302724.1094

S Atef, G Gad, S Imam, M Shawky. Ain Shams University, Cairo, Egypt

Backgrounds This prospective case control study was designed to evaluate cord blood brain derived neurotrophic factor level in full term newborns with perinatal asphyxia as a marker of central nervous system insult and predictor of severity of hypoxic ischemic encephalopathy, with follow up of its level during the reperfusion phase.

Material and Methods The study included twenty fullterm neonates with perinatal asphyxia (cases) and twenty controls. Cord blood samples were obtained at birth and peripheral blood samples at $72 \mathrm{~h}$ postnatal from cases only. Plasma brain derived neurotrophic factor level was measured using enzyme linked immunosorbent assay. The clinical severity of encephalopathy was graded based on Sarnat and Sarnat staging.

Results Cord Plasma brain derived neurotrophic factor level was significantly increased among cases compared to controls. Among cases, brain derived neurotrophic factor level at delivery and after $72 \mathrm{~h}$ significantly correlated with the severity of encephalopathy according to Sarnat staging being higher as severity increases. Brain derived neurotrophic factor level significantly increased after $72 \mathrm{~h}$ of life compared to its level at delivery among cases. Brain derived neurotrophic factor levels at delivery and at $72 \mathrm{~h}$ postnatal were predictors of severe Sarnat stage and poor outcome.

Conclusion We concluded that brain derived neurotrophic factor level as a marker of central nervous system insult is increased in full term newborns with perinatal asphyxia. It can serve as an indicator for the severity of encephalopathy and adverse outcomes.

\section{USE OF AMPLITUDE INTEGRATED ELECTROENCEPHALOGRAPHY IN NEWBORNS WITH SEVERE HYPERBILIRUBINEMIA}

doi:10.1136/archdischild-2012-302724.1095

M Chang, JH Shin. Department of Pediatrics, Chungnam National University Hospital, Daejeon, Republic of Korea

Background and Aims The spectrum of bilirubin-induced neurologic dysfunction (BIND) is very wide and the symptoms and signs may be very mild or absent. Amplitude-integrated electroencephalography (aEEG) allows continuous trend recording of cerebral function in high-risk newborns. However, published knowledge regarding correlation between aEEG and BIND remains limited.

In this study, we hypothesized that abnormal aEEG in infants with severe hyperbilirubinemia is useful for detection of asymptomatic BIND.

Methods This is a prospective observational study of newborns with severe hyperbilirubinemia in our NICU from April 2011 to December 2011. Patients were included if they were $\geq 34$ weeks gestational age (GA) at birth and their total serum bilirubin [TSB] $>20 \mathrm{mg} / \mathrm{dL}$. The aEEG was performed for 6 hours since admission, and rechecked when TSB is below $10 \mathrm{mg} / \mathrm{dL}$.

Results Fourteen infants were enrolled. Male to female was 10 to 4 . Their GA was $37.2 \pm 1.2$ weeks and their birth weight was $3,238 \pm 421 \mathrm{~g}$. Their peak TSB was $23.63 \pm 2.7 \mathrm{mg} / \mathrm{dL}$. Photopherapies were performed in all infants and exchange transfusions were also performed in 2 infants. Six out of 14 infants (42.8\%) showed abnormal aEEG findings such as discontinuity, abnormal cycling, depressed lower border and abnormal bandwidth span when their TSB were markedly elevated. However none had any noticeable symptoms or signs of neurologic dysfunction. All abnormal aEEG findings were normalized after treatment.
Conclusions Abnormal aEEG finding in infants with severe hyperbilirubinemia is useful for detection of asymptomatic BIND and can be reversible with appropriate treatment.

\section{ENIGMA OF MANAGEMENT OF SEIZURES IN HYPOXIC ISCHEMIC ENCEPHALOPATHY (HIE) - WHEN TO STOP ANTICONVULSANTS?}

doi:10.1136/archdischild-2012-302724.1096

'S Nangia, 'A Saili, ${ }^{2}$ A Garg. 'Division of Neonatology, Department of Pediatrics, ${ }^{2}$ Department of Pediatrics, Lady Hardinge Medical College and Kalawati Saran Children's Hospital, New Delhi, India

Background Although there is some agreement regarding what and when to initiate as anticonvulsant medication for seizures in HIE, there is no consensus about when to stop medication.

Objective To assess the effect of early stoppage of anticonvulsant drugs in HIE on seizure recurrence and neurological outcome.

Design/methods This prospective study enrolled neonates with HIE with non-metabolic seizures from August 2007 to July 2010. A loading dose of $20 \mathrm{mg} / \mathrm{kg}$ of phenobarbitone was used for seizure control. Additional mini-boluses of $5 \mathrm{mg} / \mathrm{kg}$ till a cumulative dose of $40 \mathrm{mg} / \mathrm{kg}$ followed by phenytoin was used if required.

Results Out of 59 neonates, $85 \%$ had cord pH below 7.2 and $83 \%$ had $\mathrm{BE}$ of -12 or higher. At birth $89 \%$ required positive pressure ventilation and $40 \%$ needed mechanical ventilation during NICU stay. 26/59(44\%) had seizure onset before $6 \mathrm{hrs}$ and 23/59 (39\%) between $6-12 \mathrm{hrs}$. $64 \%$ had a single episode of seizure, $22 \%$ had $2-3$ episodes and 10\% had 4-6 episodes. Twelve babies expired and 47 were discharged without anticonvulsant. There was no recurrence of seizure in $44 / 47(94 \%)$. On follow up at 3 months $40 / 47(85 \%)$, at 6 months $30 / 35(86 \%)$, at 12 months $25 / 29(86 \%)$, at 24 months $23 / 26(89 \%)$ and at 30 months $13 / 16(81 \%)$ had normal neurological outcome.

Conclusions This pilot work suggests that potentially apoptotic anticonvulsant drugs can probably be stopped early as soon as seizures abate in HIE without increased risk of seizure recurrence or adverse neurological outcome.

\section{ACTIVATED PROTEIN C DECREASES ENDOTOXIN-INDUCED INFLAMMATORY RESPONSES IN INFANTS WITH NEONATAL ENCEPHALOPATHY}

doi:10.1136/archdischild-2012-302724.1097

1,2,3,4 HO Eliwan, 2,3FO O'Hare, ${ }^{2} \mathrm{D}$ Sweetman, ${ }^{3} \mathrm{~W}$ Watson, ${ }^{3} \mathrm{~A}$ O'Neill, ${ }^{1,4,5,6} \mathrm{EJ}$ Molloy. ${ }^{1}$ Neonatology, Our Lady's Children's Hospital, Crumlin; ${ }^{2}$ Neonatology, National Maternity Hospital, Holles Street; ${ }^{3}$ UCD School of Medicine and Medical Science, Conway Institute for Biomolecular and Biomedical Science, University College Dublin; ${ }^{4}$ Royal College of Surgeon; ${ }^{5}$ National Maternity Hospital, Holles Street; ${ }^{6}$ UCD School of Medicine and Medical Science, Conway Institute for Biomolecular and Biomedical Science, Univeristy College Dublin, Dublin, Ireland

Introduction Infection and inflammation can be antecedents of neonatal encephaloapthy (NE) and increase the risk of neurological sequelae. Activated protein C (APC) has anticoagulant and antiinflammatory effects and provides neuroprotection in ischemic brain and spinal cord injury.

Aims To examine neutrophil and monocyte responses to Lipopolysaccharide (LPS) in infants with NE $(n=22)$ and also the effect of APC compared with healthy adult controls $(n=15)$.

Methods Whole blood was incubated with LPS +/-APC and TLR4, $\mathrm{CD} 11 \mathrm{~b}$ expression, and reactive oxygen intermediate (ROI) release from neutrophils and monocytes was examined by flow cytometry.

Results Neutrophil and monocyte CD11b expression was significantly increased in response to LPS in adults controls $(p<0.001)$ and NE infants $(p<0.001)$. However infants with NE were LPS-hyporesponsive 